\title{
COMPLICAÇÕES DE TRAUMA PÉLVICO E FEMORAL EM IDOSOS EM AMBIENTE DOMICILIAR: REVISÃO INTEGRATIVA DE LITERATURA
}

\author{
Gabriel Queiroz Bastos*1, Clezio Figueiredo Martins², Carolina Palmeira Teixeira Martins ${ }^{3}$, \\ Rodrigo Alves de Medeiros Queiroz ${ }^{4}$, Mariane Costa Santos de Tavares ${ }^{5}$, JamylleMarla de Souza \\ Santana $^{6}$, Glauber Bastos Barreto ${ }^{7}$, Gabriel Cheles Nascimento Matos ${ }^{8}$, Arnaldo Neto da Cunha \\ Bandeira $^{8}$, Ana Maria Romani ${ }^{8}$, Jorge Vinícius Leocádio Monteiro ${ }^{8}$ \\ and Iris Lorena Fernandes Santos ${ }^{9}$
}

\begin{abstract}
${ }^{1}$ Discente do Curso de Graduação em Medicina Faculdades Santo Agostinho, Vitória da Conquista - BA, Brasil; ${ }^{2}$ Médico. Cirurgião Ortopédico. Docente do Curso de Graduação em Medicina Faculdades Santo Agostinho, Vitória da Conquista -BA, Brasil; ${ }^{3}$ Médica. Infectologista. Docente do Curso de Graduação em Medicina Faculdades Santo Agostinho, Vitória da Conquista -BA, Brasil; ${ }^{4}$ Médico. Pós-Graduado em Obesidade e Sarcopenia. Trabalha pela Prefeitura Municipal de Barreiras; Clínica Therapeia em Irece-BA; ${ }^{5}$ Fisioterapeuta. Discente do Curso de Graduação em Medicina Faculdades Santo Agostinho, Vitória da Conquista - BA, Brasil; ${ }^{6}$ Médica. Especialista em Ginecologia e Obstetrícia. Clínemater Jequié-BA; ${ }^{7}$ Médico residente. Hospital do Subúrbio. Salvador - BA; ${ }^{8}$ Discente do Curso de Graduação em Medicina. Centro Universitário Aparício Carvalho - FIMCA; ${ }^{9}$ Discente do Curso de Graduação em Medicina Faculdades Santo Agostinho, Vitória da Conquista - BA, Brasil
\end{abstract}

\section{ARTICLE INFO}

\section{Article History:}

Received $07^{\text {th }}$ January, 2021

Received in revised form

$19^{\text {th }}$ February, 2021

Accepted 20 $0^{\text {th }}$ March, 2021

Published online $22^{\text {th }}$ April, 2021

\section{Key Words:}

Chave: Idoso. Ferimentos e Lesões. Lesões do Quadril. Fraturas de Fêmur.

*Corresponding author: Gabriel Queiroz Bastos

\begin{abstract}
Quedas na população idosa são referidas na literatura como causas de grande morbimortalidade. Com o avanço da idade observa-se alterações anátomo-fisiopatológicas que propiciam a perda de equilíbrio e força muscular, facilitando a ocorrência de quedas. O objetivo deste estudo foi analisar as principais complicações de trauma pélvico e femoral em idosos em ambiente domiciliar. Trata-se de uma revisão integrativa de literatura, cujas bases de dados consultadas abrangerão a Medical LiteratureAnalysis and Retrieval System Online (MEDLINE), Literatura Latino-americana e do Caribe em Ciências da Saúde (LILACS), e Scientific Electronic Library Online (SciELO), priorizando estudos originais, publicados nos últimos cinco anos (2016-2021). Os Descritores em Ciências da Saúde (DECS) utilizados para busca foram: Idoso, Ferimentos e Lesões, Lesões do Quadril, Fraturas de Fêmur e complicações.. As quedas em idosos são referidas na literatura como principais causas de trauma pélvico e femural, estando ainda correlacionadas com maior índice de hospitalizações, intervenções cirúrgicas, e óbitos. A maior proporção dos casos de traumas pélvico e femural em idosos ocorre por mecanismos de baixa energia, tendo a queda da própria altura como evento mais descrito nos estudos. Verifica-se maior ocorrência de quedas e fraturas pélvicas e femurais em mulheres, e em idosos em condição de subnutrição.
\end{abstract}

Copyright (C) 2021, Gabriel Queiroz Bastos et al, This is an open access article distributed under the Creative Commons Attribution License, which permits unrestricted use, distribution, and reproduction in any medium, provided the original work is properly cited.

Citation: Gabriel Queiroz Bastos, Clezio Figueiredo Martins, Carolina Palmeira Teixeira Martins, Rodrigo Alves de Medeiros Queiroz, Mariane Costa Santos de Tavares, Jamylle Marla de Souza Santana, Glauber Bastos Barreto, Gabriel Cheles Nascimento Matos, Arnaldo Neto da Cunha Bandeira, Ana Maria Romani, Jorge Vinícius Leocádio Monteiro and Iris Lorena Fernandes Santos, 2021. "Complicações de trauma pélvico e femoral em idosos em ambiente domiciliar: revisão integrativa de literatura", International Journal of Development Research, 11, (04), 46214-46218.

\section{INTRODUÇÃO}

Oenvelhecimento populacional é um fenômeno extremamente relevante, que vem sendo palco de intensas discussões em Saúde Pública e Social. A Organização das Nações Unidas (ONU) nomeou o período de 1975 a 2025 como a "Era do Envelhecimento", evidenciando assim a intensa transição demográfica atual, com aumento de idosos, e maior participação destes na sociedade
(TAVARES; LIMA, 2017). Tal fenômeno tem provocado mudanças na dinâmica social e econômica e invertendo a pirâmide etária. Atualmente, existem cerca de 600 milhões de indivíduos considerados idosos no mundo e estima-se que este número triplique até o ano de 2050. Aliado a isto, as mudanças de concepção, a inserção da mulher no mercado de trabalho, a redução do número de filhos, a melhoria das políticas públicas e o próprio envelhecimento da população já idosa, contribuem ainda mais para o aumento da velocidade do ritmo de transição (MOURA ; VERAS, 2017). 
Bassler et al. (2017) referem que segundo a Organização Mundial de Saúde (OMS) pessoa idosa é definida como aquela de 60 anos de idade ou mais, considerando países em desenvolvimento, e de 65 anos ou mais, considerando os países desenvolvidos. Tal definição, também foi adotada pelo Ministério da Saúde, que considera idoso o indivíduo com idade superior à 60 anos. Souza et al (2018) afirmam que o envelhecimento pode ser considerado um dos fenômenos mais importantes do século e que até o ano de 2050 todas as localidades do planeta terão $25 \%$ de suas populações composta por idosos, excetuando-se o continente africano. A sobreposição da população feminina também é um fenômeno importante, especialmente quando são observadas idades mais avançadas. Estimou-se no ano de 2012 que de 100 mulheres com idade igual ou superior a 60 anos, existiam cerca de 84 anos nas mesmas condições, e ao comparar mulheres com idade igual ou superior a 80 anos, a cada cem destas, existiam somente 61 homens. Isto pode ser explicado pelo fato de que naturalmente as mulheres vivem mais, porém, devido as relações discrepantes de gênero que afetam sociedade, estas possuem menos oportunidades de crescimento e qualidade de vida inferior quando comparado a população masculina (SOUSA et al., 2018).

$\mathrm{O}$ avanço na longevidade humana altera espantosamente o cenário demográfico mundial. Os principais fatores que determinaram o crescimento da população idosa no mundo foram a queda na taxa de natalidade, fecundidade e mortalidade. Por meio dos métodos contraceptivos, a inserção da mulher no mercado de trabalho e o maior acesso à educação e à informação determinaram a queda no número de nascimentos, que passou a ser planejado e controlado (SILVA, 2011). O progresso e a urbanização proporcionaram um salto qualitativo no nível de vida do homem, através da melhoria dos serviços de saneamento básico, da elevação do padrão nutricional e da melhoria das condições de trabalho e moradia, além das conquistas nas áreas da medicina, da saúde pública e da farmacologia, que reduziram de maneira drástica o número de mortes (MIRANDA; MENDES; SILVA, 2016). O envelhecimento da população mundial vem ocorrendo de maneira acelerada. No Brasil, de acordo com dados do Instituto Brasileiro de Geografia e Estatística (IBGE, 2018) existem aproximadamente 30,2 milhões de pessoas com idade superior ou igual a 60 anos, correspondendo à $18,6 \%$ da população total do país. O aumento da proporção de idosos em escala mundial se deve à queda das taxas de natalidade, fecundidade e mortalidade. Tal população comumente apresenta uma série de comorbidades advindas do próprio desgaste fisiológico determinado pela idade, estando comumente sujeito aos riscos de quedas (FERREIRA et al., 2012). Conforme exposto pela literatura o envelhecimento da população brasileira se deu a partir da década de 40 . Por volta do ano de 1940 a taxa de fecundidade brasileira manteve-se inalterada e houve uma queda importante nas taxas de mortalidade proporcionando uma prevalência da população jovem e com grande potencial de crescimento. Vinte anos depois, devido as mudanças na dinâmica social, as taxas de fecundidade diminuíram substancialmente, principalmente entre as populações com maior poder aquisitivo. A taxa de fecundidade no ano de 1940 era de 6,2 filhos, no ano de 1991 caiu para 2,9 filhos e no ano de 2010 para 1,9 filhos, determinando e justificando, o rápido envelhecimento da população brasileira (REIS; NORONHA ; WAJNMAN, 2016).

Diante disto, percebe que o envelhecimento é um fenômeno natural cada vez mais prevalente na sociedade e que reflete a melhoria dos serviços de saúde e a evolução da tecnologia e do desenvolvimento científico. Atrelado a isto, são exigidas mudanças sociais e econômicas que possam oferecer a oportunidade de envelhecer de forma saudável com independência e autonomia, pois, a prevalência da longevidade está intimamente relacionada com a proteção social, maiores oportunidade de emprego e melhor acesso à informação (NUNES, 2017). Além disto, o melhor acesso a saúde é um importante determinante, pois, o aumento da longevidade relacionase também com um aumento da prevalência de riscos como quedas e de outros fatores de adoecimento (MELO et al., 2017). Lima e Cezário (2014) referem que a queda é descrita como uma "Síndrome Geriatra", que é uma das principais causas de traumas em idosos em ambiente domiciliar. Os autores ponderam ainda que as quedas possuem fatores de risco intrínsecos e extrínsecos, que possuem grande impacto na condição de saúde, qualidade de vida, e funcionalidade dos idosos. Em estudo desenvolvido por Lemos (2010) os autores pontuam que as fraturas de pelve e fêmur recebem destaque entre as complicações decorridas de traumas em idosos, sendo que as fraturas de colo de fềmur são as principais causas de hospitalização do idoso, havendo ainda grande correlação com a fratura e evolução para o óbito no período de 12 meses, ou para a perda da autonomia do idoso. De acordo com Ávila, Pereira e Bochi (2015) o processo de envelhecimento, associado à presença de doenças crônico-degenerativas, bem como, condições sociais inadequadas podem propiciar a ocorrência de traumas com o idoso no ambiente domiciliar. Conforme os pesquisadores as quedas, e demais traumas externos em idosos constituem um verdadeiro problema de saúde pública, tanto por sua incidência, como pela consequente morbimortalidade entre idosos, representando ainda um fator que demanda grande gasto aos cofres públicos. As consequências dos traumas vão desde perda de confiança para deambular até receio de novos eventos, aumentando assim sua dependência, as lesões advindas da imobilidade, e a possibilidade de novas lesões. Analisando a população idosa, tem-se que as fraturas de fêmur e lesões no quadril são extremamente prevalentes em acidentes domésticos, e culminam com elevada morbimortalidade nessa população (FERREIRA et al., 2017). Diante do elevado envelhecimento da população mundial, e importância da abordagem da saúde do idoso. Buscou-se neste estudo analisar as principais complicações do trauma pélvico e femoral em idosos em ambiente domiciliar. Levando-se em consideração o contexto de envelhecimento populacional, e o impacto de traumas externos ocorridos com idosos no ambiente domiciliar, o estudo aqui proposto se justifica pela possibilidade de melhor compreender as complicações advindas de tais traumas, seus principais mecanismos de ocorrência, e ainda possíveis repercussões biopsicossociais relacionados.

\section{MATERIAIS E MÉTODOS}

O estudo proposto se caracteriza como uma revisão integrativa de literatura. Conforme referido por Souza, Silva e Carvalho (2010, p.102) a revisão integrativa de literatura "proporciona a síntese de conhecimento", sendo que seus resultados são úteis na aplicação prática dos conceitos discutidos. Foi realizada uma busca não sistemática na literatura científica. Cujas bases de dados consultadas abrangerão a Medical LiteratureAnalysis and Retrieval System Online (MEDLINE), Literatura Latino-americana e do Caribe em Ciências da Saúde (LILACS), e Scientific Electronic Library Online (SciELO), priorizando estudos originais, publicados nos últimos cinco anos (2016-2021). Os Descritores em Ciências da Saúde (DECS) utilizados para busca foram: Idoso, Ferimentos e Lesões, Lesões do Quadril, Fraturas de Fêmur e complicações. A análise dos dados se deu por abordagem qualitativa, considerando os critérios propostos por Souza, Silva e Carvalho (2010), em que se procede a interpretação e síntese dos resultados, comparando os dados coletados da análise dos artigos selecionados com o referencial teórico.

\section{RESULTADOS E DISCUSSÃO}

Após busca foram estruturadas três categorias temáticas: Processo natural de envelhecimento; Quedas em idosos; Complicações de Traumas pélvico e femural em idosos

Processo natural do envelhecimento: $O$ processo natural de envelhecimento compreende uma variedade de alterações no organismo. Estas alterações ocorrem no âmbito psicológico, morfológico, bioquímico e fisiológico. Sendo assim, levando-se em consideração as particularidades e individualidade de cada ser humano, as alterações naturais exigem também uma capacidade de adaptação ao meio ambiente, o que acaba se tornando um fator limitante com o envelhecimento (OLIVEIRA et al., 2017). Diversas são as teorias que tentam compreender o envelhecimento natural e as 
alterações ocasionadas no organismo. A teoria dos radicais livres é a mais difundida atualmente e defende que as espécies químicas liberadas no metabolismo celular são responsáveis pelo estresse oxidativo e por causar prejuízos as estruturas celulares e ao próprio DNA. Além desta, há ainda a teoria associadas ao encurtamento do telômero, que defende o fato de que as extremidades cromossômicas diminuem a tal ponto que não é mais capaz de realizar as suas funções básicas e vitais para o metabolismo celular. O processo de senescência está associado ainda a modificações na expressão de genes por meio de processos de metilação em enzimas essenciais para a manutenção do corpo (KAIM ; BACKES, 2019). Em sua natureza multifatorial, o envelhecimento pode ser acelerado por diversos fatores epigenéticos e genéticos, com importantes modificações metabólicas a nível celular e molecular. Estas alterações promovem o desequilíbrio das funções homeostáticas do organismo e intensa redução da capacidade motora e funcional do corpo. Além disto, há ainda diminuição da massa celular ativa comprometendo sistemas essenciais, como o Sistema Nervoso Central (SNC), responsável por funções biológicas internas, psíquicas, sensações e emoções. O comprometimento do SNC impacta ainda na mobilidade, postura, equilíbrio e na marcha (BANKOFF, 2019). O sistema osteoarticular, responsável pela homeostasia energética em movimento e repouso, também é significativamente comprometido com o processo de senescência. O sistema muscular quando em desuso sofre atrofia e consequente adelgaçamento de suas fibras, pois, a capacidade plástica deste tecido traduz-se na capacidade de movimentação que o corpo é capaz de realizar. Já o sistema ósseo, com a continua movimentação, é capaz de manter a sua resistência, conservar o conteúdo mineral que o compõe e ainda regenerar-se de pequenas fraturas que ocorrem em seu interior. Com o envelhecimento, o osso trabecular é o mais afetado, pois a sua manutenção depende de fatores dinâmicos, hormonais e minerais (BANKOFF, 2019). Nesta perspectiva, além dos fatores intrínsecos associados as alterações fisiológicas do organismo, há ainda os fatores extrínsecos que influenciam na progressão da fragilidade dos idosos. Entre os principais fatores extrínsecos, estão principalmente os ambientais e sociais que se associam diretamente com a dependência para a realização de atividades básicas diárias e risco de quedas nesta população. Este conjunto culmina na perda da autonomia e independência dos idosos, levando-os a consumir mais assiduamente os serviços de saúde (SOUZA et al., 2017). Diante disto, conclui-se que o envelhecimento é algo natural e previsto que ocorra com todos os indivíduos. As mudanças metabólicas, sociais, estruturais e fisiológicas que ocorrem impactam diretamente na qualidade de vida, na qualidade do sono e na realização de atividades da rotina. Por isto, é importante compreender os fatores associados a senescência para que seja possível diferenciá-los dos processos patológicos também comuns na população idosa (YAREMCHUK, 2018).

Quedas em idosos: Devido a sua imensa relevância, o envelhecimento é caracterizado como uma questão de saúde pública que é ocasionado por processos multidimensionais baseados em fatores intrínsecos e extrínsecos. Neste contexto, encarar a população idosa como sujeitos de vulnerabilidades permite um melhor delineamento das ações de prevenção e promoção a saúde, imprescindível para a implementação de políticas públicas adequadas (BARBOSA et al., 2017). Souza et al (2017) afirmam que as quedas na população idosa são um dos principais problemas que levam a hospitalização e ao óbito. Afirmam ainda que os principais fatores de risco associados a quedas estão a incapacidade funcional, a utilização de fármacos como benzodiazepínicos, hipotensão postural, a presença de ambiente inadequado e propício a episódios de quedas, doenças crônicas e histórico de quedas. Além disto, o sexo feminino é um fator de risco importante, assim como a idade avançada, déficits psicocognitivos e função neuromuscular fragilizada (SOUZA et al., 2017). Estudos apontam que as quedas em idosos são mais prevalentes em países de média e baixa renda, locais em que a população idosa se torna cada vez mais prevalente e crescendo em nível acelerado. Cerca de $80 \%$ de todas as incapacidades ocasionadas por lesões não intencionais estão associadas a episódios de quedas, tornando-se fato preocupante quando relacionado a qualidade de vida desta população. Alem disto, cerca de $10 \%$ a $15 \%$ dos idosos que vivenciam episódios de queda suportam as lesões provocadas (PORTELLA ; LIMA , 2017). No Brasil, cerca de 30\% dos idosos caem pelo menos uma vez durante o ano e cerca de dois terços ocasionam em óbito, sendo as mulheres as mais afetadas em número. Fatores ambientais como pisos, tapetes e obstáculos no chão são importantes fatores de risco para estes episódios, assim como a doença Parkinsoniana e a polifarmácia (MARQUES; OTONI; FAUSTO, 2017). Além disto, a institucionalização também é considerada um importante fator de risco para quedas em idosos. Isto ocorre pois, o idoso muda completamente seus hábitos que antes eram associados a ambiente familiar e é levado a se adaptar a novas rotinas, ambiente e a se adaptar com pessoas que não faziam parte de seu convívio. Todos estes fatores podem levar a alterações funcionais, psicológicas, cognitivas e motoras, associadas ainda ao isolamento, ao sedentarismo e ao próprio medo de cair, ocasionando assim, riscos para a sua independência e autonomia (NETO et al., 2017). Diante disto, o risco de queda em idosos podem gerar consequências irreversíveis e aumentar a prevalência de morbidade e mortalidade na população. Por isto, essencial que medidas de prevenção sejam adotadas com o intuito de reduzir a incidência de quedas e propiciar uma vida com mais qualidade e soluções acessíveis que possam se adaptar as necessidades individuais de cada um (LIMA, 2017).

Complicações de Traumas pélvico e femural em idosos: Após a ocorrência de uma queda no idoso este pode sofrer diversos tipos de fraturas, sendo que a maios comum é a ocorrência de fratura de fềmur, que podem se dar na região proximal, distal ou fraturas da diáfise femural. Após instalação da fratura, a maior parte dos idosos necessidade de correção cirúrgica, o que eleva custos em saúde e aumenta a morbimortalidade associado à queda (ARAÚJO et al., 2017). As fraturas e lesões do anel pélvico em idoso estão comumente associadas à traumas de baixa energia, sendo que a queda da própria altura é referida pela literatura como uma das principais causas desse tipo de trauma, sobretudo pela elevada porosidade óssea nessa faixa etária(PEREIRA et al., 2017). Considera-se ainda que tais lesões sejam mais comuns no sexo feminino, e o local de maior ocorrência seja no próprio domicílio do idoso e/ou instituição (DIAS et al., 2021). A ocorrência de maior número de quedas em mulheres idosas pode ter relação com fatores como a maior exposição à atividades domésticas, perda mais acentuada de massa magra, menor força muscular, assim como também maior prevalência de doenças crônicas como diabetes e hipertensão (ARAÚJO et al., 2017). Um ponto importante a ser referido é que em traumas com baixa energia, geralmente as fraturas são mais estáveis, e há menor índice de complicações quando comparado à traumas de alta energia. Contudo, no contexto do idoso, já fragilizado, e com outras comorbidades, mesmo traumas de baixo impacto podem promover elevado tempo de restrição ao leito, e agravamento do contexto de fragilidade (PEREIRA et al., 2017). Há ainda referido na literatura pesquisada a evolução de idosos após fraturas pélvicas com quadro álgico persistente, e restrição da amplitude de movimento articular, desencadeando perda da qualidade de vida, e funcionalidade. Observa-se ainda que mesmo fraturas relativamente simples em indivíduos adultos, pela porosidade óssea já descrita neste estudo, podem apresentar maior cominuição, com maior potencial de gravidade e evolução para artrose (GUIMARÃES et al., 2010). Diversos fatores podem estar associados à complicações de traumas pélvicos e femural no idoso. Em estudo realizado com 19 idosos em pós-operatório imediato para tratamento de fraturas de fêmur proximal, além de observar-se maior prevalência no sexo feminino, como já referido, foi encontrada ainda relação entre desnutrição, e maior período de internação, e infecções do sítio cirúrgico. No estudo $89,4 \%$ dos idosos que sofreram fraturas após queda de própria altura estavam com quadro de desnutrição associado, o que pode ter contribuído para ocorrência de quedas, bem como de suas complicações (DIAS et al., 2021).

Em relação às complicações perioperatórias e mortalidade em idosos submetidos à correção cirúrgica de fratura femural estudo com 182 pacientes constatou que $30,8 \%$ da amostra evoluiu para óbito até um ano após a cirurgia, sendo a causa predominante o choque séptico. A 
principal complicação, tanto pré quanto pós-operatória, foi distúrbio hidroeletrolítico. Houve ainda relato de complicações tromboembólicas, e infecção da ferida operatória (BARBOSA et al., 2019). Foi consenso entre os autores estudados que após eventos de quedas em idosos, seguido de fraturas, há complicações imediatas, perioperatórias e ainda complicações tardias, com maior morbidade associada. A redução de funcionalidade, decréscimo da qualidade de vida, e maior mortalidade são referidos com frequência pelos autores consultados (LEHTONEN et al., 2918; BARBOSA et al., 2019).

\section{CONSIDERAÇÕES FINAIS}

Tendo o processo de envelhecimento mundial como um fato, e a transição epidemiológica e demográfica como fatores determinantes de novas necessidades, torna-se fundamental identificar fragilidades e riscos na população idosa que possam promover adoecimento, perda da funcionalidade, e maior dependência de serviços de saúde e assistenciais. As quedas em idosos são referidas na literatura como principais causas de trauma pélvico e femural, estando ainda correlacionadas com maior índice de hospitalizações, intervenções cirúrgicas, e óbitos. A maior proporção dos casos de traumas pélvico e femural em idosos ocorre por mecanismos de baixa energia, tendo a queda da própria altura como evento mais descrito nos estudos. Verifica-se maior ocorrência de quedas e fraturas pélvicas e femurais em mulheres, e em idosos em condição de subnutrição.

\section{REFERÊNCIAS}

ARAÚJO, M.M.R. et al. Características dos idosos que realizaram cirurgia devido à fratura de fêmur. Rev. Enferm. Dig. Cuidado e Prom. da Saúde, v.2, n.2, 2017.

AVILA, M. A. G. de; PEREIRA, G. J. C.; BOCCHI, S. C. M.. Cuidadores informais de idosos em pós-operatório de cirurgia de fêmur proximal: prevenção de novas quedas. Ciênc. saúde coletiva, Rio de Janeiro, v. 20, n. 6, p. 1901-1907, June 2015.

BANKOFF, A. D. P..Equilibrio corporal, postura corporal no processo de envelhecimento e medida de prevenção através do exercício físico: uma revisão. Revista Saúde e Meio Ambiente - RESMA, Três Lagoas, v. 9, n. 2, p. 17-33, 2019.

BARBOSA, K. T. Fernandes et al. Envelhecimento e vulnerabilidade individual: um panorama dos idosos vinculados a estratégia de saúde da família. Texto contexto enferm., Florianópolis, v. 26, n. jun. 2017.

BARBOSA, Talita de Almeida et al . Complicações perioperatórias e mortalidade em pacientes idosos submetidos a cirurgia para correção de fratura de fêmur: estudo prospectivo observacional. Rev. Bras. Anestesiol., Campinas, v. 69, n. 6, p. 569-579, Dec. 2019.

BASSLER, T.C. et al. Avaliação da qualidade de vida de idosos residentes em Instituição de Longa permanência para idosos. Rev enferm UFPE online, v.11, n.1, p. 10-17, 2017.

BRASIL. Instituto Brasileiro de Geografia e Estatística - IBGE. Número de idosos cresce $18 \%$ em 5 anos e ultrapassa 30 milhões em 2017. Agência IBGE Notícias: Estatísticas sociais. Publicado em: 26/04/2018. Disponível em: https://agenciadenoticias.ibge.gov.br/agencia-noticias/2012agencia-de-noticias/noticias/20980-numero-de-idosos-cresce18-em-5-anos-e-ultrapassa-30-milhoes-em-2017. Acesso em 11 jun. 2020.

DIAS, Tiane Raquel da Silva et al . Avaliação do estado nutricional e correlação com complicações cirúrgicas em pacientes idosos submetidos a tratamento cirúrgico de fratura do fêmur proximal. Rev. bras. ortop., São Paulo , v. 56, n. 1, p. 104-108, Feb. 2021.

FERREIRA, A.C.F. et al. Incidência e caracterização de idosos na clínica ortopédica do Hospital Regional Cáceres por fratura de fêmur. Rev. G\&S, v.4, n.2, p.1932-41, 2017.
FERREIRA, O. G. L. et al . Envelhecimento ativo e sua relação com a independência funcional. Texto contexto - enferm., Florianópolis, v. 21, n. 3, p. 513-518, Sept. 2012.

GUIMARÃES, J.A.M. et al. Tratamento cirúrgico da fratura instável do anel pélvico em pacientes esqueleticamente imaturos. Rev. Bras. Ortop. v.45, n.6, p.583-589, 2010.

KAIM, M.; BACKES, L. T. H.. Envelhecimento celular: teorias e mecanismos. Revista Saúde Integrada, São Caetano de Sul, v. 12, n. 33, p. 178-189, 2019.

LEHTONEN, Eva Jolanda Irene et al . Tendências no tratamento cirúrgico das fraturas do colo do fêmur em idosos. Einstein (São Paulo), São Paulo, v. 16, n. 3, eAO4351, 2018 .

LEMOS, C.A.G. Lesões por causas externas e fisioterapia: estudo em um centro de reabilitação municipal de média complexidade de Uberlândia - MG. 2010. 91p. (Dissertação (Mestrado em Ciências da Saúde) - Faculdade de Medicina. Universidade Federal de Uberlândia, Uberlândia, 2010.

LIMA , F. F. O.. Perfil Sociodemográfico e nível de dependência funcional de idosos com risco de quedas. E Rev. Mult. Psic, [S. l.], v. 11, n. 39, p. 164-178, 2017.

LIMA, D.; CEZARIO, V.. Quedas em idosos e comorbidades clínicas. Revista Hospital Universitário Pedro Ernesto, [S.1.], v. 13, n. 2, mar. 2014.

MARQUES, J. M.; OTONI, D. B.; FAUSTO, L. M.. Intervenções de enfermagem para a prevenção de quedas em idosos acometidos por doença de parkinson. Revista Edu. Meio Amb. Sau., , [S. l.], v. 7, n. 2, p. 60-74, 2017.

MELO, L. A. et al. Fatores socioeconômicos, demográficos e regionais associados ao envelhecimento populacional. Rev. Bras. Geriatr. Gerontol., Rio de Janeiro, v. 20, n. 4, p. 494502, 2017.

MIRANDA, G. M. D.; MENDES, A. da C. G.; SILVA, A. L. A. da. O envelhecimento populacional brasileiro: desafios e consequências sociais atuais e futuras. Rev. bras. geriatr. gerontol., Rio de Janeiro , v. 19, n. 3, p. 507-519, June 2016 .

MOURA, Maria Martha Duque; VERAS, Renato Peixoto. Acompanhamento do envelhecimento humano em centro de convivência. Revista de Saúde Coletiva, Rio de Janeiro, v. 27, n. 1, p. 19-39, 2017.

NETO , A. H. de A. et al. Quedas em idosos institucionalizados: riscos, consequências e antecedentes. Rev. Bras. Enferm, Brasilia, v. 70, n. 4, p. 719-725, jun. 2017.

NUNES, A. M.. Demografia, envelhecimento e saúde: uma análise ao interior de Portugal. Revista Kairós Gerontologia, [S. l.], v. 20, n. 1, p. 133- 154, 2017.

OLIVEIRA, H. M. L. et al. Fisioterapia na prevenção de quedas em idosos: revisão de literatura. Revista Interdisciplinar de Estudos Experimentais, [S. l.], v. 9, p. 43- 47, 2017.

PEREIRA, G.J.C. et al. Estudo epidemiológico das fraturas e lesõesdo anel pélvico. Rev. Bras. Ortop., v.52, n.3, p.260269, 2017.

PORTELLA , M. R.; LIMA , A. P.. Quedas em idosos: reflexão sobre as políticas públicas para o envelhecimento saudável. Arq. Cienc. Saúde UNIPAR, Umuarama, v. 22, n. 2, p. 109-115, 2017.

REIS, C. S.; NORONHA, K.; WAJNMAN, S.. Envelhecimento populacional e gastos com internação do SUS: uma análise realizada para o Brasil entre 2000 e 2010. R. bras. Est. Pop., Rio de Janeiro, v. 33, n. 3, p. 591-612, 2016.

SILVA, L.M. Envelhecimento e qualidade de vida para idosos: um estudo de representações sociais. 2011. 78f. Dissertação (Mestrado em Enfermagem) - Centro de Ciências da Saúde. Universidade Federal da Paraíba, João Pessoa, 2011. Disponível em: http://www.ccs.ufpb.br/ppgeold/dissertacoes2011/luipa.pdf. Acesso em 11 mai. 2020. 
SOUSA, N. F. da S. et al. Envelhecimento ativo: prevalência e diferenças de gênero e idade em estudo de base populacional. Cad. Saúde Pública, [S. l.], v. 34, n. 11, p. 114, 2018.

SOUZA, L. H. R. et al. Queda em idosos e fatores de risco associados. Rev. Aten. Saúd, São Caetano de Sul, v. 15, n. 54, p. 55-60, 2017.

TAVARES, M.C.T.; LIMA, C.M.V. Dificuldades do idoso e familiares na medicação domiciliar. Interfaces, v.4, n.12, p. 23-31, 2017.
URSI, E.S. Prevenção de lesões de pele no perioperatório: revisão integrativa da literatura. 2005. 130 p. Dissertação (Mestrado em Enfermagem) - Escola de enfermagem de Ribeirão Preto. Universidade de São Paulo, Ribeirão Preto, 2005.

YAREMCHUK, K..SleepDisorders in theElderly. ClinGeriatrMed, v. 34, n.2, p. 205-216, 2018. 